\title{
NOVEL CURRENT-MODE QUOTIENT CIRCUIT
}

\author{
MUHAMMAD TAHER ABUELMA'ATTI \\ King Fahd University of Petroleum and Minerals, Box 203, Dhahran 31261, Saudi Arabia \\ (Received March 29, 1994; in final form May 11, 1994)
}

A novel current-mode quotient circuit is presented. The circuit uses available devices. Effects of nonideal active-elements are considered.

\section{INTRODUCTION}

In current-mode signal processing circuits the current rather than the voltage is used as the active variable either throughout the whole circuit or in certain critical areas. This can result in wider bandwidth, greater linearity, and larger dynamic range than in voltage mode circuits $[1,2]$. This justifies the large number of publications reporting various current-mode implementations (see for example [3-9]). No attempt however has been reported to implement a current-mode "one-over" circuit or more generally a current-mode quotient circuit. The transfer function of a current-mode quotient circuit is said to be ideal or accurate if the output current is linearly proportional to the quotient of two input currents. The transfer function of a current-mode "one-over" circuit is said to be ideal or accurate if "one over the output current" is linearly proportional to the input current. It is the purpose of this paper to present a current-mode quotient circuit that can also implement a current-mode "one-over" operation. These operations are very useful in a wide range of applications.

\section{PROPOSED CIRCUIT}

Consider the circuit shown in Fig. 1. Assuming that the second-generation current conveyor (CCII) is ideal with $\nu_{\mathrm{X}}=\nu_{\mathrm{Y}}, \mathrm{i}_{\mathrm{Y}}=0$ and $\mathrm{i}_{\mathrm{X}}=\mathrm{i}_{\mathrm{Z}}$, and that the operational transconductance amplifier is ideal with $\mathrm{i}_{\mathrm{d}}=\mathrm{g}_{\mathrm{m}}\left(\nu_{\mathrm{a}}-\nu_{\mathrm{b}}\right)$, where $\mathrm{g}_{\mathrm{m}}=\mathrm{I}_{1} / 2 \mathrm{~V}_{\mathrm{T}}, \mathrm{V}_{\mathrm{T}}$ is the thermal voltage and $I_{1}$ is the auxiliary bias current of the OTA, then with an ideal buffer, $\nu_{\mathrm{b}}=\nu_{\mathrm{i}}$, where $\nu_{\mathrm{b}}$ and $\nu_{\mathrm{i}}$ are the output and input voltages of the buffer, it is easy to show that the output current $I_{z}$ can be expressed as

$$
I_{z}=\frac{2 V_{T}}{R} \frac{I_{1}}{I_{2}}
$$




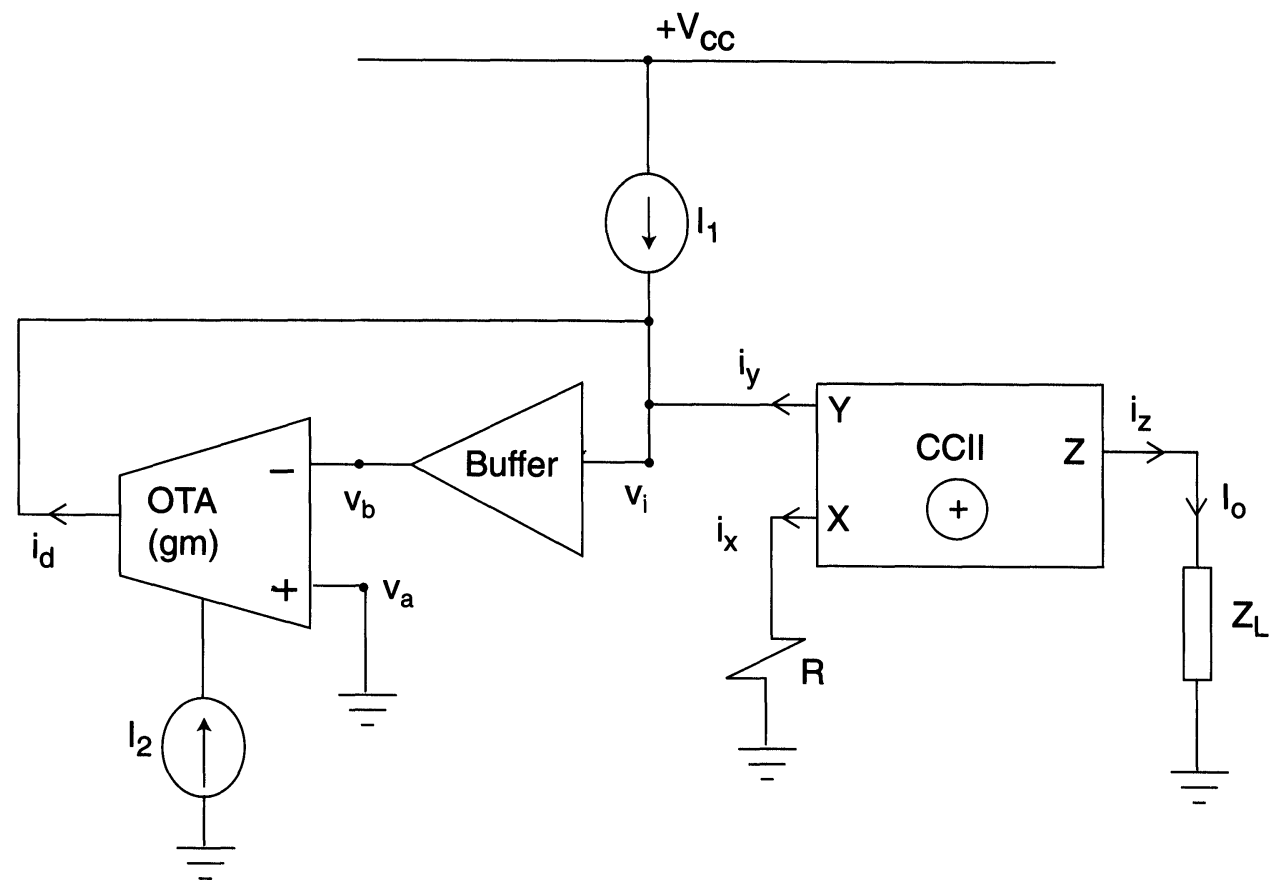

FIGURE 1 Proposed Current-Mode Quotient Circuit.

Equation (1) shows that the output current $I_{z}$ is linearly proportional to the quotient of the two input currents $I_{1}$ and $I_{2}$. Thus, the circuit of Fig. 1 can work as a currentmode quotient circuit.

Now, by obtaining the current $I_{1}$ from a constant current source, it is easy to see that the proposed circuit can work as a current-mode "one-over" circuit. Thus, a possible realization of a current-mode "one-over" circuit can be as shown in Fig. 2. This is a modified version of the bandgap voltage reference circuit [10]. Assuming that $R_{A}=R_{B}$ and an ideal operational amplifier (OA) with infinite gain, $\nu_{p}=\nu_{n}$, then

$\mathrm{I}_{\mathrm{A}}=\mathrm{I}_{\mathrm{B}}=\mathrm{V}_{\mathrm{T}} \ln (\mathrm{n}) / \mathrm{R}_{2}$

where $\mathrm{n}$ is the ratio between emitter areas of the two transistors $\mathrm{Q}_{1}$ and $\mathrm{Q}_{2}$. Thus, the current $I_{1}$ will be given by

$\mathrm{i}_{\mathrm{d}}=\left(\mathrm{I}_{\mathrm{A}}+\mathrm{I}_{\mathrm{B}}\right)$

Combining (1)-(3), routine analysis, using $\nu_{\mathrm{X}}=\nu_{\mathrm{Y}}$ and, $\mathrm{i}_{\mathrm{X}}=\mathrm{i}_{\mathrm{Z}}$ yields

$\frac{1}{\mathrm{I}_{\mathrm{o}}}=\alpha \mathrm{I}_{\mathrm{i}}$

where $\alpha=\mathrm{RR}_{2} /\left(2 \mathrm{~V}_{\mathrm{T}}\right)^{2} \ln (\mathrm{n})$. Equation (4) indicates that the realization of a current-mode "one-over" circuit is feasible. 


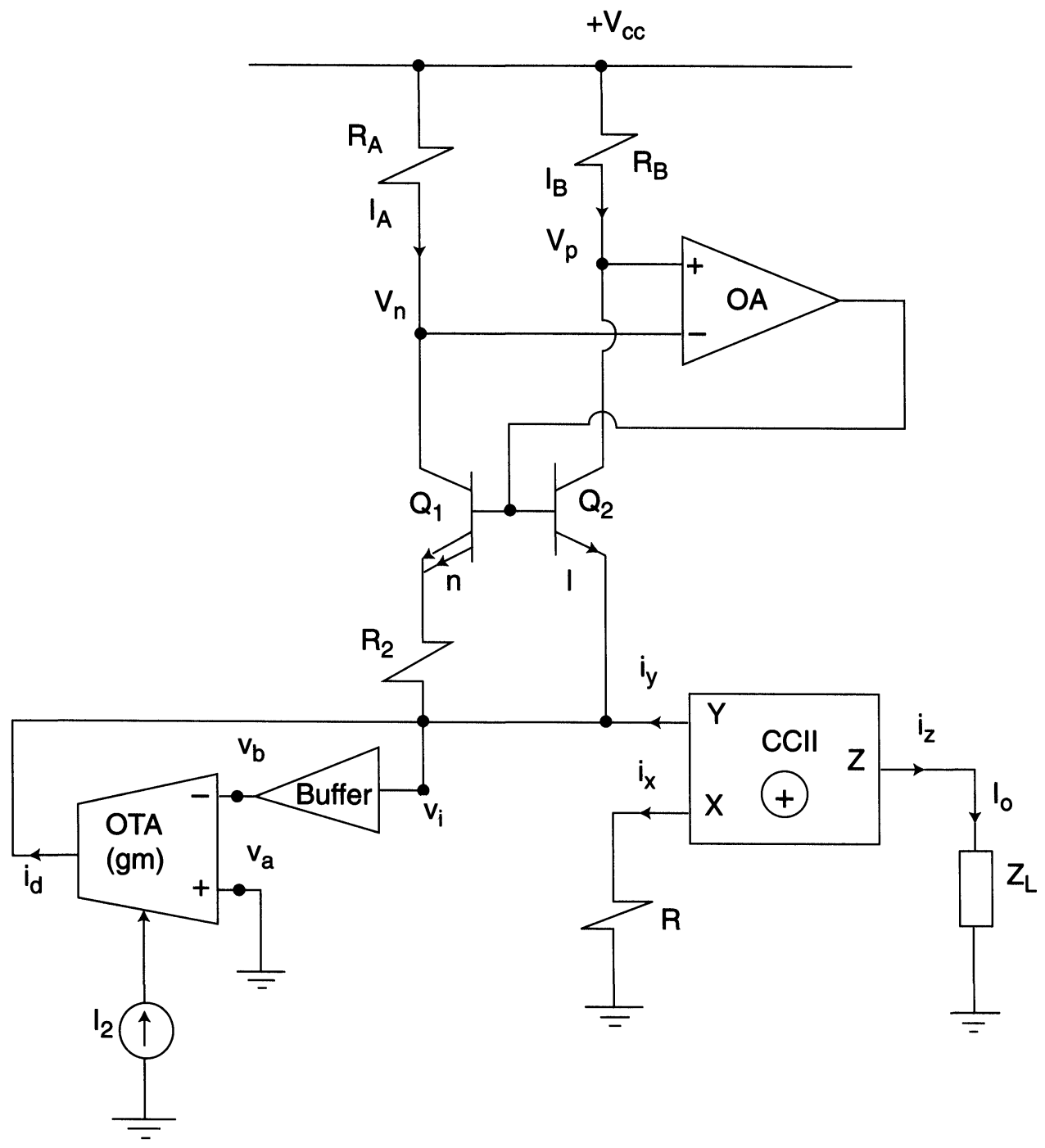

FIGURE 2 Proposed Current-Mode “One-over” Circuit.

\section{EFFECT OF NONIDEALITIES}

OAs, OTAs, buffers, and CCIIs are not ideal. Therefore, errors in the output current are expected. The finite gain of the OA may have a significant effect on the operation of the proposed circuit as $\nu_{\mathrm{p}}$ and $\nu_{\mathrm{n}}$ will be unequal and consequently the currents $I_{A}$ and $I_{B}$ will not be equal. However, proper selection of the resistors $R_{A}$ and $R_{B}$ may compensate for this effect. For example, if $\nu_{p}=(1+\delta) v_{n},|\delta| \ll$ 1 , then by selection $R_{B}=R_{A}(1-\psi),|\psi| \ll 1$, it is to show that the currents $I_{A}$ and $I_{B}$ will be equal if $\psi=\delta /\left(V_{C C} / v_{n}-1\right)$. Thus, a proper selection of the resistances $R_{A}$ and $R_{B}$ may compensate for the finite gain of the OA. 
The input impedance of the OTA may have a significant effect over the performance of the proposed circuit. Although it is possible to predict that influence, it is more practical to buffer the OTA input as shown in Fig. 1. Buffers may be non-ideal, however. A non-ideal buffer with slight gain deviation can be described by $\nu_{\mathrm{b}}=(1-\lambda) \nu_{\mathrm{i}}$, where $\lambda \ll 1$. The effect of the non-ideal CCII characteristic on the performance of the circuit of Fig. 1 can be studied using the simplified model shown in Fig. 3. This simplified model was deduced from [11]. Due to the high values of $R_{Y}$ and $R_{Z}$ and low values of $C_{Y}$ and $C_{Z}$, the effects, that have to be considered are principally due to $\phi_{\mathrm{i}}, \phi_{\nu}$, and $\mathrm{r}_{\mathrm{XY}}$. Assuming that the current transfer ratio and the voltage gain of a non-ideal CCII can be described by $\phi_{\mathrm{i}}=$ $\mathrm{i}_{\mathrm{Z}} / \mathrm{i}_{\mathrm{X}}, \phi_{\nu}=\nu_{\mathrm{X}} / \nu_{\mathrm{Y}}$ where $\phi_{\mathrm{i}}=1+\varepsilon_{\mathrm{i}},\left|\varepsilon_{\mathrm{i}}\right| \ll 1$ and $\phi_{\nu}=1+\varepsilon_{\nu},\left|\varepsilon_{\nu}\right| \ll 1$, reanalysis of the circuit of Fig. 1 yields the modified output current $I_{\tilde{o}}$

$\frac{1}{\mathrm{I}_{\mathrm{o}}^{\sim}}=\alpha\left(1+\frac{\mathrm{r}_{\mathrm{XY}}}{\mathrm{R}}\right)(1-\lambda)\left(1+\varepsilon_{\mathrm{i}}\right)\left(1+\varepsilon_{\nu}\right) \mathrm{I}_{\mathrm{i}}$

Since the parasitic resistance $r_{X Y}$ is of the order of 10-100 Ohm [12], and if we choose $r_{X Y} \ll R$, then after neglecting second-order terms, (5) reduces to

$\frac{1}{\mathrm{I}_{\mathrm{o}}^{\sim}}=\alpha\left(1+\frac{\mathrm{r}_{\mathrm{XY}}}{\mathrm{R}}-\lambda+\varepsilon_{\mathrm{i}}+\varepsilon_{\nu}\right) \mathrm{I}_{\mathrm{i}}$

Equation (6) shows that the output current will be affected by the buffer and CCII non-idealities. However, the error term in $(6),\left(1+\frac{\mathrm{r}_{\mathrm{XY}}}{\mathrm{R}}-\lambda+\varepsilon_{\mathrm{i}}+\varepsilon_{\nu}\right)$, contains positive and negative terms and cancellation of the effects of CCII and buffer nonidealities is, therefore, feasible. Thus, if $\mathrm{r}_{\mathrm{XY}} / \mathrm{R}+\varepsilon_{\mathrm{i}}+\varepsilon_{\nu}=\lambda$, (6) reduces to (4); that is, the input current-output current characteristic of the proposed "one-over" circuit is unchanged even with a non-ideal buffer and CCII. Moreover, by proper selection of the resistors $R_{A}$ and $R_{B}$, the effect of the finite gain of the operational amplifier can be compensated. Therefore, an ideal "one-over" transfer function can be realised even with a non-ideal operational amplifier, buffer, operational transconductance amplifier, and second-generation current conveyor.

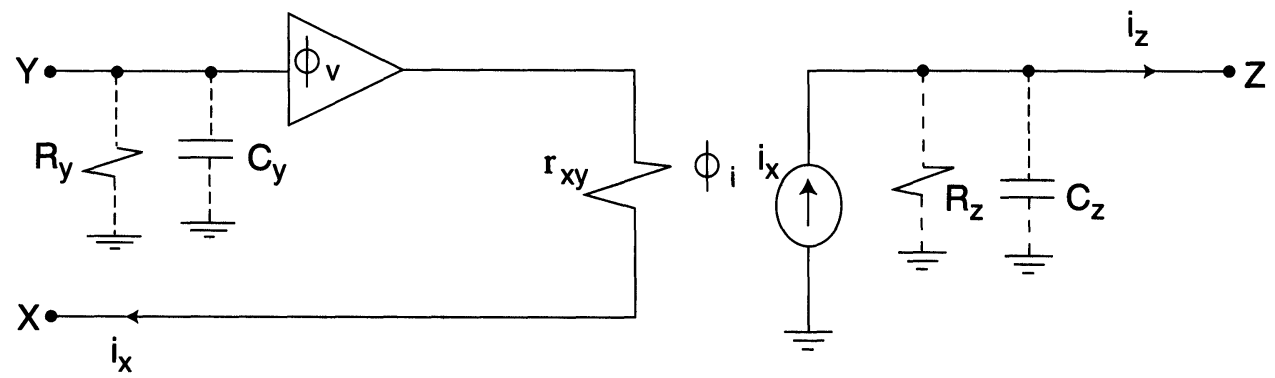

FIGURE 3 Simplified model of CCII + . 


\section{CONCLUSION}

A simple current-mode quotient and "one-over" circuit has been presented. By proper selection of the parameters, the effects of a non-ideal operational amplifier, buffer, operational transconductance, and second-generation current conveyor may cancel each other. Thus, the realization of an ideal current-mode "one-over" transfer function is feasible, even with non-ideal active components. It is worth mentioning here that the influence of temperature on $\mathrm{g}_{\mathrm{m}}$ of commercial OTAs like CA3080 can be compensated by using temperature-compensated OTAs [13-14]. Also, implementations using CMOS OTAs makes feasible the use of relatively large input voltage swings [15-17]. Thus, the major limitations of commercial OTAs like CA3080 can be avoided.

\section{REFERENCES}

1. B. Wilson, Recent developments in current conveyors and current-mode circuits, Proceedings IEE, Vol. 137, Part G, 1990, pp. 63-70.

2. G.W. Roberts and A.S. Sedra, All current-mode frequency selective circuits, Electronics Letters, Vol. 25, 1989, pp. 759-761.

3. P. Aronhime, D. Nelson and C. Adams, Applications of a first-generation current conveyor in current-mode circuits, Electronics Letters, Vol. 26, 1990, pp. 1456-1457.

4. A. Fabre, F. Martin and M. Hanafi, Current mode allpass/notch and bandpass filters with reduced sensitivities, Electronics Letters, Vol. 26, 1990, pp. 1495-1496.

5. M. Higashimura and Y. Fukui, Realization of current mode all-pass networks using a current conveyor, IEEE Transactions on Circuits and Systems, Vol. 37, 1990, pp. 660-661.

6. S.-I. Liu, H.-W. Tsao and J. Wu, Cascadable current-mode single CCII biquads, Electronics Letters, Vol. 26, 1990, pp. 2005-2006.

7. P. Aronhime and A. Dinwiddie, Biquadratic current-mode filters using a single CCI, International Journal of Electronics, Vol. 70, 1991, pp. 1063-1071.

8. P. Aronhime, D. Nelson, J. Zurada and C. Adams, Realization of current-mode complex pole all-pass networks using a single current conveyor, Proceedings IEEE International Conference on Circuits and Systems, IEEE New York, 1990, pp. 3193-3196.

9. M.T. Abuelma'atti, New current-mode-active filters employing current conveyors, International Journal of Circuit Theory and Applications, Vol. 21, 1993, pp. 93-99.

10. A.B. Grebene, Bipolar and MOS Analog Integrated Circuit Design, John Wiley \& Sons, New York, 1984.

11. B. Wilson, Performance analysis of current conveyors, Electronics Letters, Vol. 25, 1989, pp. 1596-1598.

12. B. Wilson, Current mode circuit: Analysis and CAD modelling, International Symposium on Circuits and Systems, Vol. 4, 1990, pp. 3242-3245.

13. H.S. Malvar and M. Leuttgen, Temperature compensation of OTA-based filters and amplifiers, Electronics Letters, Vol. 23, 1987, pp. 890-891.

14. M.T. Ahmed, I.A. Khan and T. Parveen, Wide range electronically tunable component multipliers, International Journal of Electronics, Vol. 65, 1988, pp. 1007-1011.

15. K.D. Pterson and R.L. Geiger, CMOS OTA structures with improved linearity, Proceedings 27 th Midwest Symposium of Circuits and Systems, 1984, pp. 63-66.

16. A. Nedungadi and T.R. Viswanathan, Design of linear CMOS transconductance elements, IEEE Transactions on Circuits and Systems, Vol. CAS-31, 1984, pp. 891-894.

17. R.R. Torrance, T.R. Viswanathan and J.V. Hanson, CMOS voltage to current transconductors, IEEE Transactions on Circuits and Systems, Vol. CAS-32, 1985, pp. 1097-1104. 

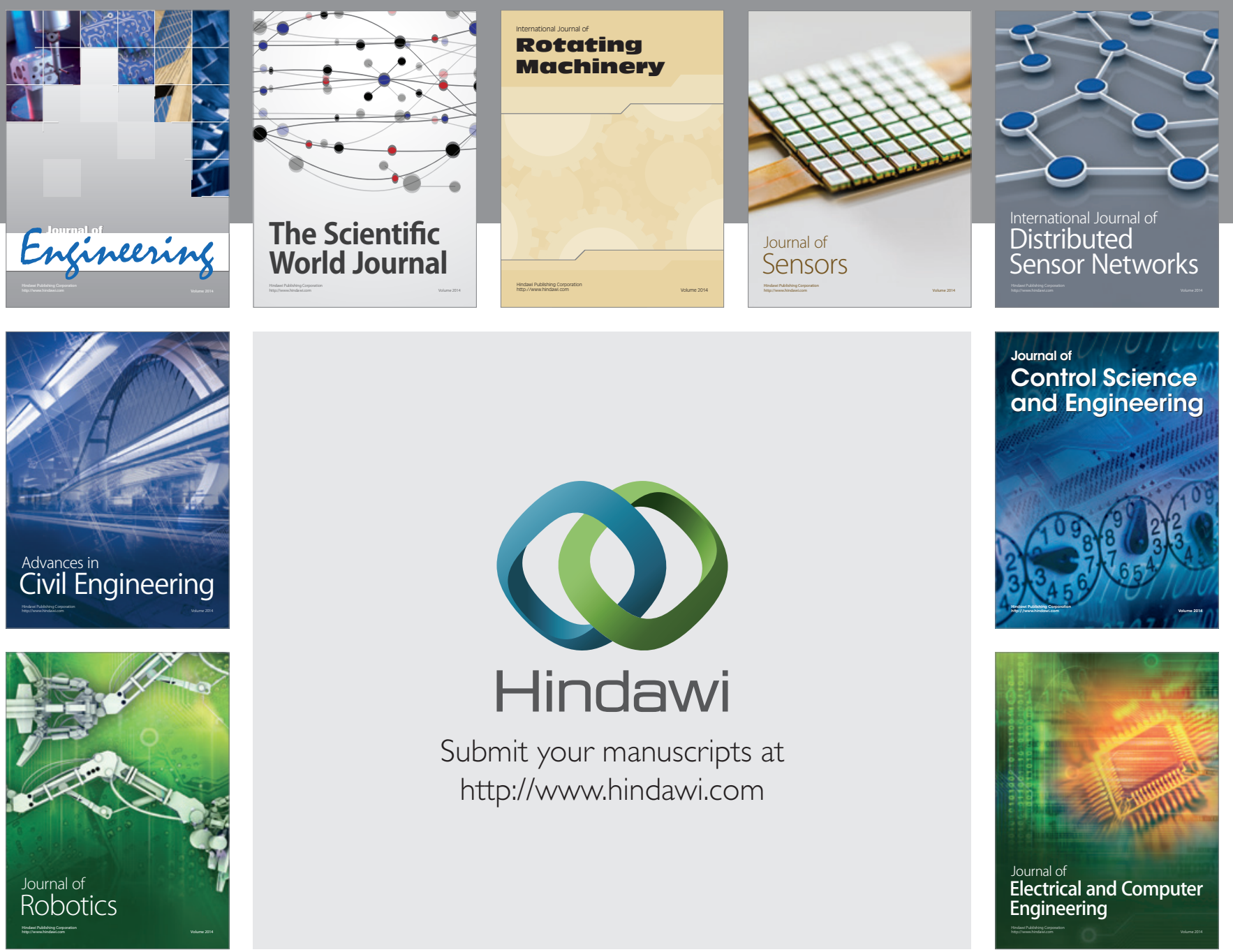

Submit your manuscripts at

http://www.hindawi.com
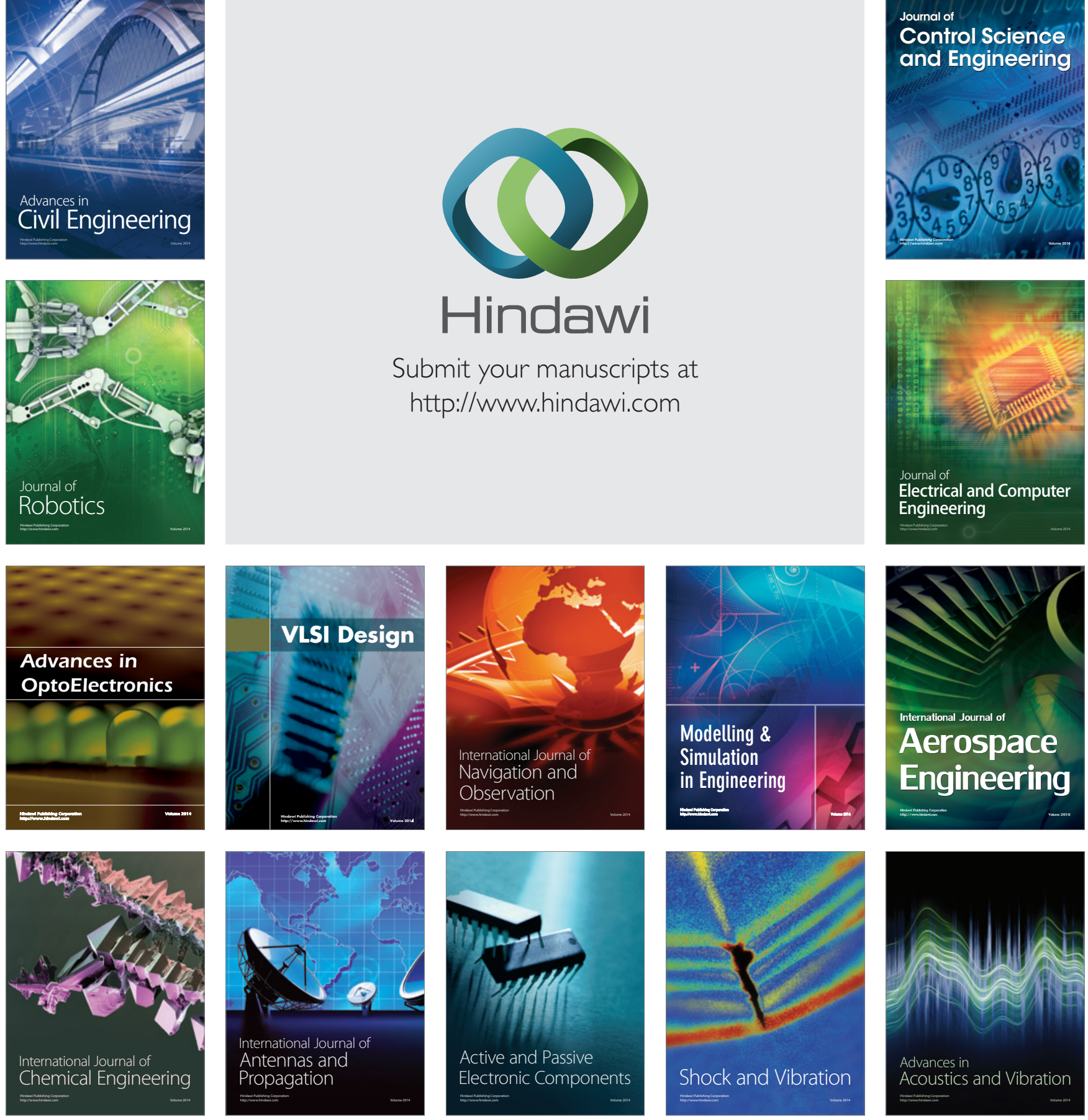